\title{
Particle number fluctuations in a non-ideal pion gas
}

\author{
Evgeni E. Kolomeitsev ${ }^{1, a}$, Maxim E. Borisov², and Dmitry N. Voskresensky²,3 \\ ${ }^{1}$ Matej Bel University, Banska Bystrica, Slovakia \\ ${ }^{2}$ National Research Nuclear University (MEPhl), Moscow, Russia \\ ${ }^{3}$ Joint Institute for Nuclear Research (JINR), Dubna, Russia
}

\begin{abstract}
We consider a non-ideal hot pion gas with the dynamically fixed number of particles in the model with the $\lambda \phi^{4}$ interaction. The effective Lagrangian for the description of such a system is obtained by dropping the terms responsible for the change of the total particle number. Within the self-consistent Hartree approximation, we compute the effective pion mass, thermodynamic characteristics of the system and identify a critical point of the induced Bose-Einstein condensation when the pion chemical potential reaches the value of the effective pion mass. The normalized variance, skewness, and kurtosis of the particle number distributions are calculated. We demonstrate that all these characteristics remain finite at the critical point of the Bose-Einstein condensation. This is due to the non-perturbative account of the interaction and is in contrast to the ideal-gas case.
\end{abstract}

\section{Introduction}

Multitudes of pions are produced in central heavy-ion collisions at RHIC and LHC energies [1]. The measured pion spectra have an approximately exponential form for pion transverse momenta $p_{T} \lesssim(1-2) m_{\pi}$, where $m_{\pi}$ is the pion, mass but show an enhancement at low transverse momenta, cf. [2]. Thus on one hand one may assume that pions form a hot expanding fireball characterized by the temperature $T(\vec{r}, t)$ and density $n(\vec{r}, t)$, on the other hand the low- $p_{T}$ enhancement can be signal of a non-vanishing chemical potential [3, 4] or a contribution from resonance decays [5], or both effects. The kinetic freeze-out temperature is estimated to be $T_{\text {kin }} \sim 100-120 \mathrm{MeV}$. An estimate [6] shows that at $T \sim 120-140 \mathrm{MeV}$ processes of the pion absorption are rather suppressed, whereas re-scattering processes are still important. Thus, one may assume that the total pion number is dynamically fixed on a time scale between a chemical freeze out, when the total pion number becomes frozen and the thermal freeze-out, when the momentum distributions stop changing [7]. The existing estimates for the typical pion density in the pion fireball are contradictory $[8,9]$ yielding values varying in a broad interval, for LHC from $n \sim 0.8$ to $2.5 n_{0}$, where $n_{0}$ is the nuclear saturation density. Already first estimates $[3,4]$ have demonstrated that experimental low- $p_{T}$ pion yields in collisions at $200 \mathrm{AGeV}$ can be fitted if the pion chemical potential $\sim 120-130 \mathrm{MeV}$ is introduced. Recent work [8] evaluated the non-equilibrium chemical potential of pions as $\mu \simeq 134.9 \mathrm{MeV}$, being very close to the critical one $\left(\mu=m_{\pi} \simeq 135-140 \mathrm{MeV}\right)$ for a Bose-Einstein condensation (BEC) in an

a e-mail: Evgeni.Kolomeitsev@umb.sk 
ideal pion gas. Non-equilibrium supercooling effects [10-12], decays of resonances [13], or a decay of the transient BEC of gluons or glueballs pre-formed at an initial stage in a heavy-ion collision, cf. [14-18] may provide effective mechanisms to drive the pion system to the Bose-Einstein condensation (BEC). Reference [10] studied a possibility of the pion BEC in the interacting relativistic pion gas with a $\lambda \varphi^{4}$ interaction under the assumption that the number of pions created in the course of ultrarelativistic heavy-ion collisions is (dynamically) fixed in the time interval between the chemical and thermal freeze-outs. The most central collisions with high pion multiplicity were proposed as the most preferable to observe effects of the pion BEC. Utilizing the Weinberg Lagrangian, the investigation of the pion BEC was continued in [19, 20]. Reference [12] demonstrated that before the formation of the BEC the interacting pion gas in ultrarelativistic heavy-ion collisions may pass several stages, including a wave-turbulence stage, effects studied in [21] in relation to the BEC in application to non-relativistic gaseous systems.

A growth of fluctuations near the critical point of the second-order and first-order phase transitions is a general property manifested in various critical opalescence phenomena, cf. [22]. References $[23,24]$ returned to consideration of the ideal pion gas and argued for the divergence of the normalized variance in the critical point of the BEC in the thermodynamic limit. The result was supported by the analysis in the micro-canonical approach. On the experimental side an enhancement of the normalized variance was observed in the high pion multiplicity events in $p p$ collisions in the energy range 50-70 GeV [25]. Here, a care should be taken, when one compares naive theoretical expectations for thermal fluctuation characteristics with actual measurements, which incorporate background contributions, the dependence on center-of-mass energy, other dynamical effects, collision centrality, kinematic cuts, etc.

In this contribution we will compute characteristics of particle number fluctuations in the nonideal hot pion gas with the dynamically fixed number of particles considered in thermodynamical limit.

\section{Effective Lagrangian for pions with dynamically fixed particle number}

As a model for the description of the pion system we use the simplified Lagrangian [26]

$$
\mathcal{L}=\frac{1}{2}(\partial \vec{\varphi})^{2}-\frac{1}{2} m_{\pi}^{2} \vec{\varphi}^{2}-\frac{1}{4} \lambda \vec{\varphi}^{4}
$$

with $\lambda \sim 1$. Indeed, comparing with the leading terms of the Weinberg Lagrangian we find $\lambda=$ $m_{\pi}^{2} / 2 f_{\pi}^{2} \simeq 1.13$, where $f_{\pi}=93 \mathrm{MeV}$ is the weak pion decay constant. The isospin vector $\vec{\varphi}=$ $\left(\varphi_{1}, \varphi_{2}, \varphi_{3}\right)$ is associated with the fields of the positive $\left(\pi^{+}\right)$, negative $\left(\pi^{-}\right)$and neutral $\left(\pi^{0}\right)$ pions $\pi^{ \pm}=$ $\frac{1}{\sqrt{2}}\left(\varphi_{1} \pm i \varphi_{2}\right), \pi^{0}=\varphi_{3}$. Positive and negative pions, being introduced as particles and anti-particles, are described by the one complex field, $\left(\pi^{-}\right)^{\dagger}=\pi^{+}$, where $(\ldots)^{\dagger}$ means the Hermitian conjugation. Neutral pions, as the self-conjugated particles, are represented by the real field $\left(\pi^{0}\right)^{\dagger}=\pi^{0}$.

The 4-point interaction among the fields $\vec{\varphi}$ included in the Lagrangian (1) modifies the pion properties and the pion-pion interaction in medium. The resulting in-medium retarded pion propagator is determined by the Dyson equation

$$
G(\omega, k)=G_{0}(\omega, k) /\left(1-G_{0}(\omega, k) \Pi(\omega, k)\right)=\longleftarrow=-+\bigcirc+\bigcirc+\ldots
$$

where the thin line is the free pion retarded propagator $G_{0}(\omega, k)=1 /\left(\omega^{2}-k^{2}-m_{\pi}^{2}+i 0\right)$, and $\omega$ and $k$ stand for the frequency and momentum of the pion, and $\Pi$ is the full pion retarded polarization 
operator determined by the diagrams shown in (2). The first diagram in (2) is the "tadpole" diagram and the second one is the "sandwich" diagram.

Now we will consider a pion system with the dynamically fixed particle number. To do this we turn in the Lagrangian (1) to the new complex fields $\varphi_{-}, \varphi_{+}, \varphi_{0}$ corresponding to pions with the positive frequencies $\pi^{-}=\varphi_{-}+\varphi_{+}^{\dagger}, \pi^{+}=\varphi_{+}+\varphi_{-}^{\dagger}$, and $\pi^{0}=\varphi_{0}+\varphi_{0}{ }^{\dagger}$. In a system with the fixed and, in general, different numbers of pions of each species the particle-anti-particle symmetry is lost, and therefore, it is possible that $\varphi_{-} \neq \varphi_{+}^{\dagger}$, and neutral pions are described by a complex field $\varphi_{0}$. In the second quantization new fields of $\pi^{-}, \pi^{+}, \pi^{0}$ mesons possess the following operator representations in the quasi-particle approximation $\hat{\varphi}_{i}=\sum_{\vec{k}} \hat{a}_{\vec{k}}^{(i)} Z_{k}^{(i)} e^{-i \omega_{i}(k) t+i \vec{k} \vec{r}}, \quad i=+,-, 0$. We choose positive solutions of the dispersion relations, $\omega_{ \pm 0}(k)>0$, and, in general case, $\omega_{-}(k) \neq \omega_{+}(k) \neq \omega_{0}(k)$ with $Z_{k}^{-} \neq Z_{k}^{+} \neq Z_{k}^{0}$. The operators $\hat{a}, \hat{b}$ and $\hat{c}$ define now the numbers of pions of the corresponding sorts $\sum_{\vec{k}} 2 \omega_{i} Z_{k}^{(i)} \overline{\hat{a}_{\vec{k}}^{(i) \dagger} \hat{a}_{\vec{k}}^{(i)}}=N_{i}$, where $\overline{(\ldots)}$ denotes averaging over the vector of state of the quantum system with the Gibbs weight factor. The mean value $\overline{\hat{a}_{\vec{k}}^{(i) \dagger} \hat{a}_{\vec{k}}^{(i)}}$, is determined by the Bose distribution with frequencies $\omega_{i}$ and chemical potentials $\mu_{i}$ fixing the particle numbers $N_{i}$.

Replacing new fields in the Lagrangian (1), we are able to separate terms, which correspond to the particle number conservation. In terms of the new fields the Lagrangian density (1) is

$$
\begin{aligned}
\mathcal{L} & =\mathcal{L}_{\text {fix }}+\mathcal{L}^{\prime}, \\
\mathcal{L}_{\text {fix }} & =\mathcal{L}_{\pi^{-}}+\mathcal{L}_{\pi^{+}}+\mathcal{L}_{\pi^{0}}+\mathcal{L}_{\pi^{+} \pi^{-} \pi^{0}}, \quad \mathcal{L}^{\prime}=\mathcal{L}_{2 \leftrightarrow 2}+\mathcal{L}_{3 \leftrightarrow 1} .
\end{aligned}
$$

Here the terms $\mathcal{L}_{\pi^{i}}=\left|\partial \varphi_{i}\right|^{2}-m_{\pi}^{2}\left|\varphi_{i}\right|^{2}-\lambda\left|\varphi_{i}\right|^{4}$ describe the interaction of pions of a certain sort and we neglected a difference in pion masses for various species, the term

$$
\mathcal{L}_{\pi^{+} \pi^{-} \pi^{0}}=-4 \lambda\left|\varphi_{-}\right|^{2}\left|\varphi_{+}\right|^{2}-2 \lambda\left(\left|\varphi_{+}\right|^{2}+\left|\varphi_{-}\right|^{2}\right)\left|\varphi_{0}\right|^{2}
$$

describes the interaction of the different pion species with each other via reactions $\pi^{+} \pi^{-} \leftrightarrow \pi^{+} \pi^{-}$and $\pi^{ \pm} \pi^{0} \leftrightarrow \pi^{ \pm} \pi^{0}$. These processes conserve the total number of pions and also the number of pions of each sort. Note that the pion self-interaction contained in the terms $\mathcal{L}_{\text {fix }}$ gives a contribution to the pionic polarization operator already in a first-order in the coupling constant $\lambda$. Such a contribution is depicted by the tadpole diagram in (2). The part of the Lagrangian density

$$
\mathcal{L}_{2 \leftrightarrow 2}=-\lambda\left(\varphi_{+} \varphi_{-} \varphi_{0}^{\dagger 2}+\varphi_{0}^{2} \varphi_{+}^{\dagger} \varphi_{-}^{\dagger}\right)
$$

corresponds to the processes $\pi^{0} \pi^{0} \leftrightarrow \pi^{+} \pi^{-}$, which, while keeping the total number of pions fixed, change the relative fractions of the pion species so that the chemical potentials obey the relation $2 \mu_{0}=$ $\mu_{+}+\mu_{-}$. For the case of the isospin-symmetric pion gas, that we will focus on in this contribution, $\mu_{0}=\mu_{+}=\mu_{-} \equiv \mu$. The term containing non-equal numbers of the pion creation and annihilation operators, $\mathcal{L}_{3 \leftrightarrow 1}=\mathcal{L}\left(\varphi_{i}^{\dagger} \varphi_{j} \varphi_{k} \varphi_{l} ; \varphi_{i} \varphi_{j}^{\dagger} \varphi_{k}^{\dagger} \varphi_{l}^{\dagger}\right), \quad i, j, k, l=+,-, 0$, corresponds to the processes with a change of the number of pions in the system, $\pi \leftrightarrow \pi \pi \pi$. These processes bring the system to the chemical equilibrium. The kinetic equation for the pion gas allowing for these reactions has the Bose distribution with vanishing chemical potential as an equilibrium solution.

The terms $\mathcal{L}_{2 \leftrightarrow 2}$ and $\mathcal{L}_{3 \leftrightarrow 1}$ give contributions to the pion polarization operator only in the second order of the coupling constant. They are represented by a sandwich diagram in (2) with different pion species in the internal lines allowed by charge conservation. The direction of the internal lines takes also into account the distinct processes: $\pi \pi \leftrightarrow \pi \pi$ (two lines are directed to the one side and one line to the other side) and $\pi \leftrightarrow \pi \pi \pi$ (all lines go from the left to the right). For $T \lesssim m_{\pi}$ of our interest the real part of the sandwich diagram proves to be small in comparison with the tadpole 
contribution. Thereby we omit the former contribution. The imaginary part of the sandwich diagram determines the rates of rescattering and absorption/production reactions. As we have discussed, the processes $\pi \pi \leftrightarrow \pi \pi$ responsible for the thermal equilibration occur essentially faster then processes $\pi \leftrightarrow \pi \pi \pi$ responsible for the chemical equilibration at $T \lesssim m_{\pi}$. Therefore, we drop furthermore the term $\mathcal{L}_{3 \leftrightarrow 1}$ of the Lagrangian, introducing instead the non-vanishing pionic chemical potentials fixing the pion numbers determined by reactions $\pi^{+} \pi^{-} \leftrightarrow \pi^{0} \pi^{0}$. Doing so we assume that these reactions are operative on the time scale of the pion fireball expansion up to its break up. Being armed by the effective Lagrangian (3) describing the system with the fixed total particle number and an arbitrary electric charge, we can study its properties.

\section{Pion spectrum in self-consistent Hartree approximation}

Varying the Lagrangian (3) with respect to $\varphi_{+}^{\dagger}, \varphi_{-}^{\dagger}, \varphi_{0}^{\dagger}$ fields we obtain a system of coupled non-linear equations of motion. For isospin-symmetric system under consideration $\varphi_{+}^{\dagger}=\varphi_{-}^{\dagger}=\varphi_{0}^{\dagger} \equiv \varphi$ and we have only one equation of motion. We solve this equation within the self-consistent Hartree approximation, which is rather appropriate at temperatures of our interest, $T \lesssim m_{\pi}$. Within this approximation the behaviour of a certain particle is determined by an averaged interaction with surrounding particles, which form a thermal bath. The properties of particles in the bath are, in turn, determined by the same equation of motion as that for the given pion (cf. bold line in (2)).

Formally, we represent the field $\varphi$ as a superposition of some picked-out field modes $\tilde{\varphi}$ and an environmental field $\xi, \varphi \rightarrow \tilde{\varphi}+\xi$. Then we keep in the equation of motion only the terms that are linear in the $\tilde{\varphi}$ and quadratic in $\xi$ (other terms vanish after averaging). As the result we find equation of motion for the field $\tilde{\varphi}$ in the Hartree approximation

$$
\partial^{2} \tilde{\varphi}+m_{\pi}^{2} \tilde{\varphi}+\Pi \tilde{\varphi}=0
$$

with the spectrum

$$
\omega(k)=\sqrt{k^{2}+m^{* 2}}, \quad m^{* 2}=m_{\pi}^{2}+\Pi,
$$

where the polarization operator

$$
\Pi=10 \lambda \overline{\left|\xi^{2}\right|}, \quad \overline{|\xi|^{2}}=\left\langle\frac{f}{2 \omega}\right\rangle \equiv \int \frac{\mathrm{d}^{3} k}{(2 \pi)^{3}} \frac{f(k)}{2 \omega(k)}, \quad f(k)=\frac{1}{e^{(\omega(k)-\mu) / T}-1},
$$

and the total pion density $n=3\langle f\rangle$. The non-commutativity of the creation-annihilation operators produces divergent contributions of quantum fluctuations to the macroscopic characteristics of the system, e.g. in (7), which have to be renormalized by subtraction of the corresponding vacuum values. The remaining finite contributions from quantum fluctuations are proved to be rather small and we omit them.

When the temperature decreases up to the value $T_{c}^{(\text {ind) }}$ there appears an "induced" BEC. It occurs for $\mu=m^{*}$. Following [20], we say about induced BEC, since the BEC could also occur in a first-order phase transition at a higher temperature, when $\mu$ reaches the free pion mass, $m_{\pi}$, if it were energetically favorable. Any first-order phase transition requires an extra time and may occur only in rare events. Therefore, we will ignore this possibility in our study here.

The dependence of the effective pion mass $m^{*}$ and the chemical potential $\mu$ on the temperature in the interacting pion gas for the isospin-symmetric matter is shown on the left panel in figure 1 . On the middle panel we demonstrate the critical temperature of the induced BEC as a function of the pion gas density. We see that the effective pion mass is larger than the free pion mass and increases with a decrease of the temperature and an increase of $\lambda$. Also the mass increases with an increase of the density. The critical temperature $T_{c}^{\text {ind }}$ for the interacting pion gas is smaller than that for the ideal gas. 

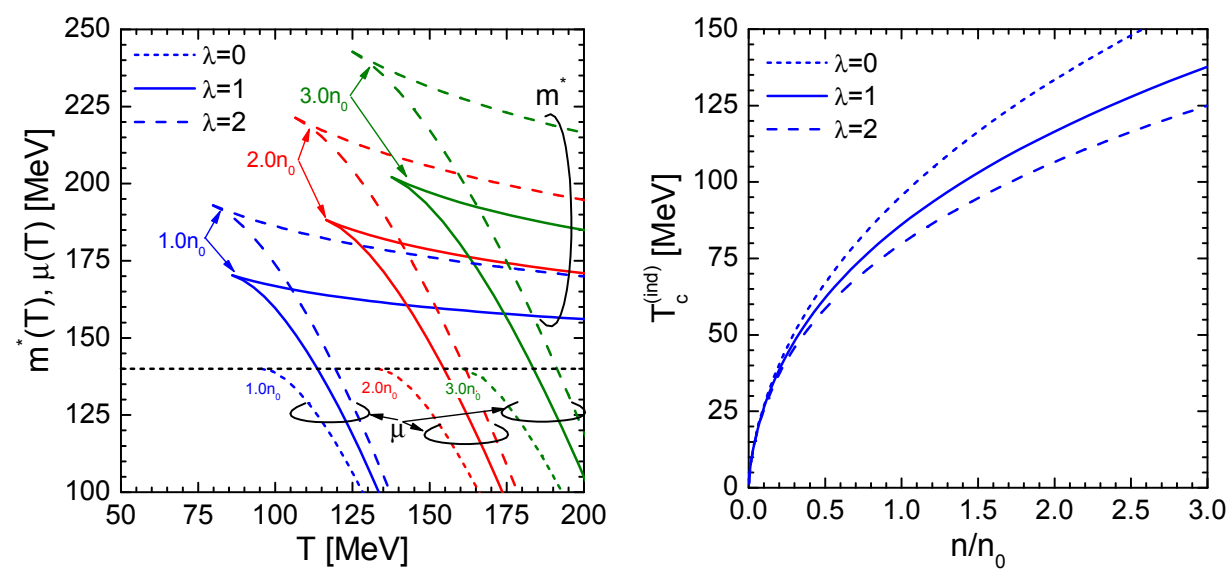

Figure 1. Left panel: effective pion mass and chemical potential in the isospin-symmetric pion gas as functions of temperature for three values of the pion density $n=n_{0}, 2 n_{0}$, and $3 n_{0}$ (here $n_{0}=0.16 \mathrm{fm}^{-3}$ ) and for the interaction parameter $\lambda=0$ (short-dash lines), $\lambda=1$ (solid lines) and $\lambda=2$ (long-dash lines). Horizontal short-dash line depicts the free pion mass. Middle panel: the critical temperature of the BEC for the isospin-symmetric pion gas as a function of the pion density for $\lambda=0,1,2$.

\section{Particle number fluctuations}

In the case of a uniform system, fluctuation characteristics of the particle number distribution can be related to susceptibilities [27]

$$
\chi_{n}=T^{n-1} \frac{\partial^{n} P}{\partial \mu^{n}},
$$

so normalized variance $(w)$, skewness $S$, and kurtosis $(\kappa)$ can be written as follows

$$
w=\frac{\chi_{2}}{\chi_{1}}, \quad S \cdot \sigma=\frac{\chi_{3}}{\chi_{2}}, \quad \kappa \cdot \sigma^{2}=\frac{\chi_{4}}{\chi_{2}},
$$

where $\sigma=\chi_{2}$ is the variance of the particle number distribution. Taking the derivative with respect to $\mu$ we should take into account the implicit dependence of $m^{*}$ on it. The first-order susceptibility is simply the particle density $\chi_{1}=\partial P / \partial \mu=n$. The susceptibilities of the second and third orders can be still presented in rather compact form

$$
\begin{aligned}
& \chi_{2}=w n=3\langle f(1+f) \mathcal{R}\rangle, \\
& \chi_{3}=3\left[\left\langle f(1+f)(2 f+1) \mathcal{R}^{3}\right\rangle+3 \frac{T}{4}\left(\frac{\partial m^{* 2}}{\partial \mu}\right)^{2}\left\langle\frac{f(1+f)}{\omega^{3}} \mathcal{R}\right\rangle-3 \frac{T^{2}}{8}\left(\frac{\partial m^{* 2}}{\partial \mu}\right)^{3}\left\langle\frac{f}{\omega^{5}}\right\rangle\right],
\end{aligned}
$$

where

$$
\mathcal{R}=1-\frac{1}{2 \omega} \frac{\partial m^{* 2}}{\partial \mu}, \quad \frac{\partial m^{* 2}}{\partial \mu}=10 \lambda \frac{\partial}{\partial \mu}\left\langle\frac{f}{2 \omega}\right\rangle\left[1-10 \lambda \frac{\partial}{\partial m^{* 2}}\left\langle\frac{f}{2 \omega}\right\rangle\right]^{-1}
$$

The expression for $\chi_{4}$ is too lengthy to be given here in a full from. For the ideal gas, $\lambda \rightarrow 0$ we have $\frac{\partial m^{* 2}}{\partial \mu} \rightarrow 0$ and $\mathcal{R} \rightarrow 0$ and the susceptibilities diverges for $T \rightarrow T_{c}^{(\text {ind) }}$ when $\mu \rightarrow m^{*}$ and 

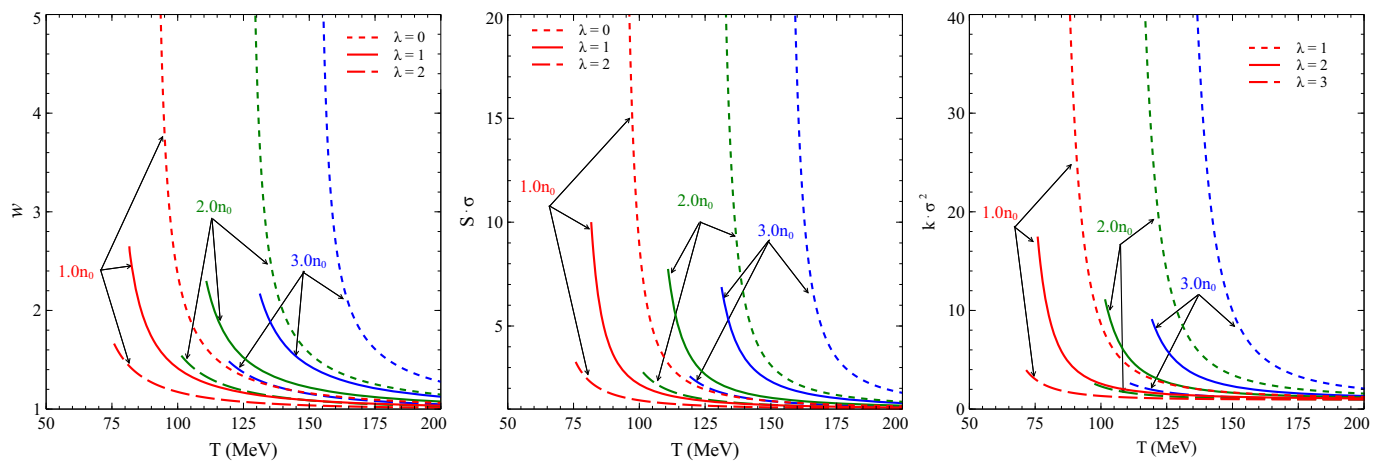

Figure 2. Normalized variance (left panel), skewness (middle panel) and kurtosis (right panel) of the pion number distributions (9) for interacting pion gas (for $\lambda=1,2)$ and for the ideal pion gas $(\lambda=0)$ as a function of temperature for various particle number densities.

the momentum integrals are determined by small momenta with $f \rightarrow \frac{2 m^{*} T_{c}^{(\text {ind })}}{2 m^{*}\left(m^{*}-\mu\right)+k^{2}}$. Then we find $\chi_{n \geq 2}^{(\lambda=0)} \propto m^{3}(T / m)^{n} /\left(1-\mu / m^{*}\right)^{n-3 / 2}$. When the interaction is taken into account in a self-consistent way (Hartree approximation our case) this behaviour changes dramatically since the factor $\mathcal{R}$ vanishes at $T \rightarrow T_{c}^{(\text {ind })}$ as

$$
\mathcal{R} \sim \frac{2}{5} \frac{m^{*}}{\lambda\left\langle f / k^{2}\right\rangle} \rightarrow \frac{2^{5 / 2} \pi}{\lambda} \frac{m^{*}}{T_{c}^{(\text {ind })}}\left(1-\mu / m^{*}\right)^{1 / 2} .
$$

We see that this vanishing of $\mathcal{R}$ compensates exactly the divergencies in (10) and (11). The sam mechanism works also for susceptibilities of higher orders. For normalized variance and skewness we find

$$
\left.w\right|_{T_{c}^{\text {ind }}}=\frac{6 m^{* 2} T_{c}^{\text {(ind) }}}{5 \lambda n}+O\left(\lambda^{0}\right),\left.\quad S \sigma\right|_{T_{c}^{\text {(ind) }}}=\frac{16}{25} \frac{\pi^{2} m^{*}}{\lambda^{2} T_{c}^{\text {(ind) }}}+O\left(\lambda^{-1}\right) .
$$

Presence of poles at $\lambda \rightarrow 0$ in these expressions indicates the non-perturbative character of our results.

In figure 2 we show the normalized variance, skewness and kurtosis of the pion number (9) for the interacting pion gas (for $\lambda=1$ and 2) and for the ideal gas $(\lambda=0)$ as functions of a temperature for three values of the density. For the ideal gas the normalized variance diverges at the critical temperature of the BEC in agreement with the statement of [24]. Therefore, the authors [24] suggested that the strong enhancement of the normalized variance can be considered as a clear signature of the BEC although effects of the finite volume smear the singularity. On the contrary, we see that for the interacting gas even in the infinite volume limit the singularity disappears provided the interaction is taken into account self-consistently (within the self-consistent Hartree approximation in our current study).

Finally, we would like to remind that the comparison of the idealized thermal fluctuations with those appeared in real experiments is in any case quite indirect because of many additional effects not taken into account, like a finite volume, realistic pion-pion interaction, the dependence on the collision energy, collision centrality, kinematic cuts, and other dynamical effects. Thus, we may only say that, if a significant growth of various pion number fluctuation characteristics were observed, it could be associated with a proximity of the system to the pion BEC either at the chemical freeze-out or at the 
thermal freeze-out, depending on the specifics of the measurements. Measurements of the normalized variance and higher moments like sckewness and kurtosis in pre-selecting high multiplicity events are desirable in order to observe a manifestation of the signatures of the Bose-Einstein pion condensation in heavy-ion collisions.

Acknowlegments. The reported study was funded by the Russian Foundation for Basic Research (RFBR) according to the research project No 16-02-00023-A and supported by the Ministry of Education and Science of the Russian Federation (Basic part), Slovak Grant No. VEGA-1/0348/18 and by THOR the COST Action CA15213.

\section{References}

[1] Nayak T K 2012 Pramana 79719.

[2] Abelev B et al. [ALICE Collaboration] 2013 Phys. Rev. C 88044910.

[3] Kataja M and Ruuskanen P V 1990 Phys. Lett. B 243181.

[4] Mishustin I N, Satarov L N, Maruhn J, Stöcker H and Greiner W 1992 Phys. Lett. B 276403.

[5] J. Sollfrank, P. Koch, and U. Heinz, Z. Phys. C 52 (1991) 593.

[6] Goity J L and Leutwyler H 1989 Phys. Lett. B 228517.

[7] Gerber P, Leutwyler H and Goity J L 1990 Phys. Lett. B 246513.

[8] Begun V, Florkowski W and Rybczynski M 2014 Phys. Rev. C 90054912.

[9] Teaney D 2002 Preprint nucl-th/0204023.

[10] Voskresensky D N 1994 J. Exp. Theor. Phys. 78793 [Zh. Eksp. Teor. Fiz. 105 1473].

[11] Voskresensky D N, Blaschke D, Röpke G and Schulz H 1995 Int. J. Mod. Phys. E 41.

[12] Voskresensky D N 1996 Phys. Atom. Nucl. 592015 [Yad. Fiz. 59 2090].

[13] Ornik U, Plümer M and Strottmann D 1993 Phys. Lett. B 314401.

[14] J.P. Blaizot, F. Gelis, J.F. Liao, L. McLerran, and R. Venugopalan, Nucl. Phys. A 873 (2012) 68.

[15] Z. Xu, K. Zhou, P. Zhuang, and C. Greiner, Phys. Rev. Lett. 114 (2015) 182301.

[16] N. Kochelev, Phys. Part. Nucl. Lett. 13 (2016) 149.

[17] A. Peshier and D. Giovannoni, J. Phys. Conf. Ser. 668 (2016) 012076.

[18] N. Tanji and R. Venugopalan, Phys. Rev. D 95 (2017) 094009.

[19] Kolomeitsev E E and Voskresensky D N, 1995 Phys. Atom. Nucl. 582082 [Yad. Fiz. 58 2195].

[20] Kolomeitsev E E, Kämpfer B and Voskresensky D N 1996 Acta Phys. Polon. B 273263.

[21] Semikoz D V and Tkachev I I 1995 Phys. Rev. Lett. 74 3093;

Semikoz D V and Tkachev I I 1997 Phys. Rev. D 55489.

[22] Landau L D and E. M. Lifshitz E M 1984 Electrodynamics of Continuous Media vol 8 (Oxford: Pergamon press).

[23] Begun V V and Gorenstein M I 2007 Phys. Lett. B 653190.

[24] Begun V V and Gorenstein M I 2008 Phys. Rev. C 77064903.

[25] Kokoulina E 2012 Prog. Theor. Phys. Suppl. 193, 306;

Kokoulina E [SVD-2 Collaboration] 2013 PoS ICHEP 2012259.

[26] Itzykson C and Zuber J B 1980 Quantum Field Theory (New York: McGraw-Hill).

[27] Asakawa M and Kitazawa M 2016 Prog.Part.Nucl.Phys. 90, 299. 